\title{
The existence of solutions to a class of boundary value problems with fractional difference equations
}

\author{
Yuanyuan Pan ${ }^{1}, Z_{\text {Zhenlai Han }}{ }^{*}$, Shurong Sun ${ }^{1,2}$ and Chuanxia Hou'
}

\author{
"Correspondence: \\ hanzhenlai@163.com \\ 'school of Mathematical Sciences, \\ University of Jinan, Jinan, Shandong \\ 250022, P.R. China \\ Full list of author information is \\ available at the end of the article
}

\begin{abstract}
In this paper, we study the existence and uniqueness of solutions for the boundary value problem of fractional difference equations

$$
\left\{\begin{array}{l}
-\Delta^{v} y(t)=f(t+v-1, y(t+v-1)), \\
y(v-3)=0, \quad \Delta y(v-3)=0, \quad y(v+b)=g(y)
\end{array}\right.
$$

and

$$
\left\{\begin{array}{l}
-\Delta^{v} y(t)=\lambda f(t+v-1, y(t+v-1)), \\
y(v-3)=0, \quad \Delta y(v-3)=0, \quad y(v+b)=g(y),
\end{array}\right.
$$

respectively, where $t=1,2, \ldots, b, 2<v \leq 3, f:\{v-1, \ldots, v+b\} \times \mathbb{R} \rightarrow \mathbb{R}$ is a continuous function and $g \in C\left([v-3, v+b]_{\mathbb{Z}_{v-3}}, \mathbb{R}\right)$ is a continuous functional. We prove the existence and uniqueness of a solution to the first problem by the contraction mapping theorem and the Brouwer theorem. Moreover, we present the existence and nonexistence of a solution to the second problem in terms of the parameter $\lambda$ by the properties of the Green function and the Guo-Krasnosel'skii theorem. Finally, we present some examples to illustrate the main results.
\end{abstract}

MSC: $34 \mathrm{~A} 08 ; 34 \mathrm{~B} 18 ; 39 \mathrm{~A} 12$

Keywords: discrete fractional equation; boundary value problem; existence and uniqueness of solution; fixed point theorem; eigenvalue

\section{Introduction}

In recent years, fractional differential equations have been of great interest. It is caused both by the intensive development of the theory of fractional calculus itself and by the applications of such constructions in various sciences such as physics, mechanics, chemistry and engineering. Mathematicians have employed this fractional calculus in recent years to model and solve a variety of applied problems. Indeed, as Podlubny outlines in [1], fractional calculus aids significantly in the fields of viscoelasticity, capacitor theory, electrical circuits, electro-analytical chemistry, neurology, diffusion, control theory and statistics.

The continuous fractional calculus has developed greatly in the last decades. Some of the recent progress in the continuous fractional calculus includes the paper [2], in which the authors explored a continuous fractional boundary value problem of conjugate type 
using cone theory, they then deduced the existence of one or more positive solutions. Of particular interest with regard to the present paper is the recent work by Benchohra et al. [3]. In that paper, the authors considered a continuous fractional differential equation with nonlocal conditions. Other recent work in the direction of those articles may be found, for example, in [4-12].

In recent years, a number of papers on the discrete fractional calculus have appeared, such as [13-30], which has helped to build up some of the basic theory of this area. For example, Atici and Eloe discussed the properties of the generalized falling function, a corresponding power rule for fractional delta-operators and the commutativity of fractional sums in [13]. They presented in [13] more rules for composing fractional sums and differences. Goodrich studied a two-point fractional boundary value problem in [16], which gave the existence results for a certain two-point boundary value problem of right-focal type for a fractional difference equation. At the same time, a number of papers appeared, and these began to build up the theoretical foundations of the discrete fractional calculus. For example, a recent paper by Atici and Eloe [14] explored some of the theories of a conjugate discrete fractional boundary value problem. Discrete fractional initial value problems were considered in a paper by Atici and Eloe [15].

Atici and Eloe in [14] considered a two-point boundary value problem for the finite fractional difference equation

$$
\begin{aligned}
& -\Delta^{v} y(t)=f(t+v-1, y(t+v-1)), \quad t=1,2, \ldots, b+1, \\
& y(v-2)=0, \quad y(v+b+1)=0,
\end{aligned}
$$

where $1<v \leq 2$ is a real number, $b \geq 2$ is an integer and $f:[v, v+b]_{\mathbb{N}_{v-1}} \times \mathbb{R} \rightarrow \mathbb{R}$ is continuous. They analyzed the corresponding Green function, provided an application and obtained sufficient conditions for the existence of positive solutions for a two-point boundary value problem for a nonlinear finite fractional difference equation.

Goodrich in [18] considered a discrete fractional boundary value problem of the form

$$
\begin{aligned}
& -\Delta^{v} y(t)=f(t+v-1, y(t+v-1)), \\
& y(v-2)=0, \quad y(v+b)=g(y),
\end{aligned}
$$

where $t \in[0, b]_{\mathbb{N}_{0}}:=\{0,1, \ldots, b\}, f:[v-2, v-1, \ldots, v+b-1]_{\mathbb{N}_{v-2}} \times \mathbb{R} \rightarrow \mathbb{R}$ is a continuous function, $g \in C\left([v-2, v+b]_{\mathbb{Z}_{v-3}}, \mathbb{R}\right)$ is a given functional, and $1<v \leq 2$. He established the existence and uniqueness of a solution to this problem by the contraction mapping theorem, the Brouwer fixed point theorem and the Guo-Krasnosel'skii fixed point theorem.

Although the boundary value problem of fractional difference equations has been studied by several authors, the present works are almost all concerned with $1<\mu \leq 2$, very little is known in the literature about a fractional difference equation with $2<\mu \leq 3$.

Motivated by all the works above, in this paper, we first aim to study the following boundary value problem:

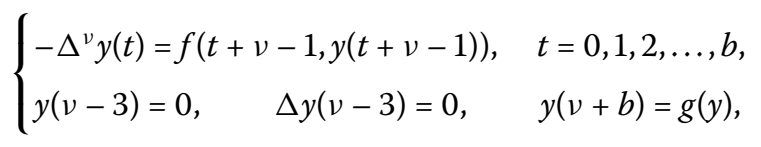


where $t=0,1,2, \ldots, b, 2<v \leq 3, f:\{v-1, \ldots, v+b-1\}_{\mathbb{N}_{v-1}} \times \mathbb{R} \rightarrow \mathbb{R}$ is continuous and $g \in C\left([v-3, v+b]_{\mathbb{Z}_{v-3}}, \mathbb{R}\right)$ is a continuous functional.

Our second aim is to investigate the boundary value problem of a fractional difference equation with parameter

$$
\begin{cases}-\Delta^{v} y(t)=\lambda f(t+v-1, y(t+v-1)), & t=0,1,2, \ldots, b, \\ y(v-3)=0, \quad \Delta y(v-3)=0, & y(v+b)=g(y),\end{cases}
$$

where $t=0,1,2, \ldots, b, 2<v \leq 3, f:\{v-1, \ldots, v+b-1\}_{\mathbb{N}_{v-1}} \times \mathbb{R} \rightarrow \mathbb{R}$ is continuous and $g \in C\left([v-3, v+b]_{\mathbb{Z}_{v-3}}, \mathbb{R}\right)$ is a continuous functional, $\lambda$ is a positive parameter. We establish some sufficient conditions for the nonexistence and existence of at least one or two positive solutions for the boundary value problem by considering the eigenvalue intervals of the nonlinear fractional differential equation with boundary conditions.

The plan of this paper is as follows. We first give the form of solutions of problem (1.1), second we prove the existence and uniqueness of a solution to problem (1.1) by the contraction mapping theorem and the Brouwer theorem, and then the eigenvalue intervals for the boundary value problem of nonlinear fractional difference equation (1.2) are considered by the properties of the Green function and the Guo-Krasnosel'skii fixed point theorem on cones. Finally we present some examples to illustrate the main results.

\section{Preliminaries}

For the convenience of the readers, we first present some useful definitions and fundamental facts of fractional calculus theory, which can be found in [13, 14].

Definition 2.1 [14] We define $t^{\underline{\nu}}:=\frac{\Gamma(t+1)}{\Gamma(t+1-v)}$ for any $t$ and $v$, for which the right-hand side is defined. We also appeal to the convention that if $t+1-v$ is a pole of the gamma function and $t+1$ is not a pole, then $t^{\underline{\nu}}=0$.

Definition 2.2 [14] The $v$ th fractional sum of a function $f$, for $v>0$, is defined by

$$
\Delta^{-v} f(t ; a):=\frac{1}{\Gamma(v)} \sum_{s=a}^{t-v}(t-s-1)^{\frac{v-1}{f}} f(s)
$$

for $t \in\{a+v, a+v+1, \ldots\}:=\mathbb{N}_{a+v}$. We also define the $v$ th fractional difference for $v>0$ by $\Delta^{v} f(t):=\Delta^{N} \Delta^{\nu-N} f(t)$, where $t \in \mathbb{N}_{a+v}$ and $v \in \mathbb{N}$ is chosen so that $0 \leq N-1<v \leq N$.

Lemma 2.1 [13] If $t \leq r$, then $t^{\underline{\alpha}} \leq r^{\underline{\alpha}}$ for any $\alpha>0$.

Lemma 2.2 [14] Let $0 \leq N-1<v \leq N$. Then $\Delta^{-v} \Delta^{v} y(t)=y(t)+C_{1} t^{\frac{\nu-1}{1}}+C_{2} t^{\frac{\nu-2}{2}}+\cdots+$ $C_{N} t^{\underline{\nu-N}}$ for some $C_{i} \in \mathbb{R}$ with $1 \leq i \leq N$.

Lemma 2.3 [17] For $t$ and $s$, for which both $(t-s-1)^{\underline{v}}$ and $(t-s-2)^{\underline{v}}$ are defined, we find that

$$
\Delta_{s}\left[(t-s-1)^{\underline{v}}\right]=-v(t-s-2)^{\underline{v}-1} .
$$


Now let us consider a linear boundary value problem, which is important for us to facilitate the analysis of problems (1.1) and (1.2).

Lemma 2.4 Let $h:[v-1, v+b-1]_{\mathbb{Z}_{\nu-3}} \rightarrow \mathbb{R}$ and $g: \mathbb{R}^{b+4} \rightarrow \mathbb{R}$. A function $y$ is a solution of the problem

$$
\begin{aligned}
& -\Delta^{v} y(t)=h(t+v-1), \\
& y(v-3)=0, \\
& \Delta y(v-3)=0, \\
& y(v+b)=g(y),
\end{aligned}
$$

where $t \in[0, b]_{\mathbb{Z}_{0}}$ if and only if $y(t), t \in[v-3, v+b]_{\mathbb{Z}_{v-3}}$, has the form

$$
y(t)=\sum_{s=0}^{b} G(t, s) h(s+v-1)+\frac{t^{\underline{\nu-1}}}{(v+b) \underline{\underline{v-1}}} g(y),
$$

where

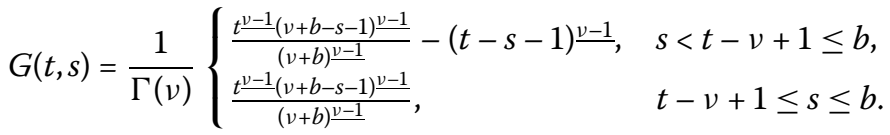

Proof By Lemma 2.2, we obtain

$$
y(t)=-\Delta^{-v} h(t+v-1)+C_{1} t^{\frac{\nu-1}{2}}+C_{2} t^{\frac{\nu-2}{2}}+C_{3} t^{\frac{\nu-3}{}}, \quad t \in[v-3, v+b]_{\mathbb{Z}_{v-3}} .
$$

From (2.2) we get

$$
y(v-3)=-\left.\Delta^{-v} h(t+v-1)\right|_{t=v-3}+C_{1}(v-3)^{\frac{v-1}{v}}+C_{2}(v-3)^{\frac{v-2}{w}}+C_{3}(v-3)^{\frac{v-3}{v}} .
$$

Noting that

$$
\begin{aligned}
& \left.\Delta^{-v} h(t+v-1)\right|_{t=v-3}=-\left.\frac{1}{\Gamma(v)} \sum_{s=0}^{t-v}(t-s-1)^{\frac{\nu-1}{}} h(s+v-1)\right|_{t=v-3}=0, \\
& (v-3) \frac{v-1}{v}=0, \quad(v-3)^{\frac{v-2}{v}}=0,
\end{aligned}
$$

we deduce $C_{3}=0$.

By (2.3) we have

$$
\Delta y(v-3)=y(v-2)-y(v-3)=0,
$$

then $y(v-2)=0$. We note that

$$
y(v-2)=-\left.\Delta^{-v} h(t+v-1)\right|_{t=v-2}+C_{1}(v-2)^{\frac{v-1}{2}}+C_{2}(v-2)^{\frac{v-2}{2}}
$$


and

$$
\left.\Delta^{-v} h(t+v-1)\right|_{t=v-2}=0, \quad(v-2)^{\frac{v-1}{}}=0,
$$

it follows that $C_{2}=0$.

From (2.4) we know

$$
y(v+b)=-\left.\Delta^{-v} h(t+v-1)\right|_{t=v+b}+C_{1}(v+b)^{\frac{v-1}{2}}=g(y),
$$

which implies that

$$
C_{1}=\frac{1}{(v+b) \frac{v-1}{\Gamma(v)}}\left[\frac{1}{s=0} \sum^{b}(v+b-s-1)^{\frac{v-1}{2}} h(s+v-1)+g(y)\right] .
$$

Consequently, we deduce that $y(t)$ has the form

$$
\begin{aligned}
& y(t)=-\frac{1}{\Gamma(v)} \sum_{s=0}^{t-v}(t-s-1)^{\frac{v-1}{}} h(s+v-1) \\
& +\frac{1}{(v+b) \underline{v-1}}\left[\frac{1}{\Gamma(v)} \sum_{s=0}^{b}(v+b-s-1)^{\frac{v-1}{h}} h(s+v-1)+g(y)\right] t^{\underline{v-1}} \\
& =-\frac{1}{\Gamma(v)} \sum_{s=0}^{t-v}(t-s-1)^{\frac{v-1}{h}} h(s+v-1)
\end{aligned}
$$

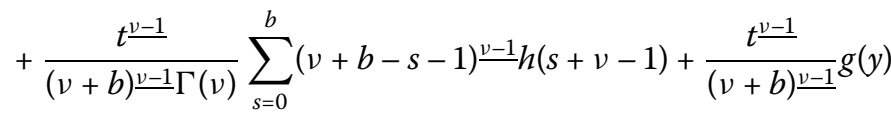

$$
\begin{aligned}
& =\sum_{s=0}^{b} G(t, s) h(s+v-1)+\frac{t \underline{v-1}}{(v+b) \underline{v-1}} g(y)
\end{aligned}
$$

for $t \in[v-3, v+b]_{\mathbb{Z}_{v-3}}$, where

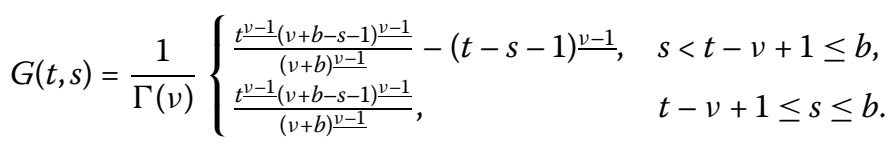

This shows that if (2.1)-(2.4) has a solution, then it can be represented by (2.7) and that every function of the form (2.7) is a solution of (2.1)-(2.4), which completes the proof.

Theorem 2.1 The Green function $G(t, s)$ satisfies the following conditions:

(i) $G(t, s)>0$ for $(t, s) \in[v-3, v+b]_{\mathbb{Z}_{v-3}} \times[0, b]$.

(ii) $\max _{t \in[v-3, v+b]_{\mathbb{Z}_{v-3}}} G(t, s)=G(s+v-1, s)$ for $s \in[0, b]$.

(iii) There exists a positive number $\gamma \in(0,1)$ such that

$$
\min _{t \in\left[\frac{b+v}{4}, \frac{3(b+v)}{4}\right]} G(t, s) \geq \gamma \max _{t \in[v-3, v+b]_{\mathbb{Z}_{v-3}}} G(t, s)=\gamma G(s+v-1, s) \quad \text { for } s \in[0, b] .
$$

The proof of this theorem is similar to that of Theorem 3.2 in [14]. Hence, we omit the proof here. 


\section{Existence and uniqueness of solution}

In this section, we wish to show that under certain conditions, problem (1.1) has at least one solution. We know that problem (1.1) can be recast as an equivalent summation equation. It follows from Lemma 2.4 that $y$ is a solution of (1.1) if and only if $y$ is a fixed point of the operator $T: \mathbb{R}^{b+4} \rightarrow \mathbb{R}$, where

$$
\begin{aligned}
(T y)(t):= & -\frac{1}{\Gamma(v)} \sum_{s=0}^{t-v}(t-s-1)^{\frac{v-1}{}} f(t+v-1, y(t+v-1)) \\
& +\frac{t \frac{v-1}{(v+b) \frac{v-1}{\omega} \Gamma(v)} \sum_{s=0}^{b}(v+b-s-1)^{\frac{v-1}{}} f(t+v-1, y(t+v-1))}{} \\
& +\frac{t \frac{v-1}{(v+b) \frac{v-1}{2}}}{(v(y)}
\end{aligned}
$$

for $t \in[v-3, v+b]_{\mathbb{Z}_{v-3}}$. We use this fact to prove the first existence theorem.

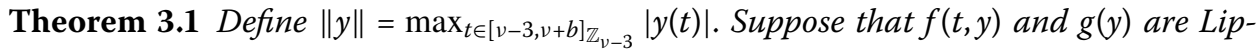
schitz in $y$. That is, there exist $\alpha, \beta>0$ such that $\left|f\left(t, y_{1}\right)-f\left(t, y_{2}\right)\right| \leq \alpha\left\|y_{1}-y_{2}\right\|, \mid g\left(y_{1}\right)-$ $g\left(y_{2}\right) \mid \leq \beta\left\|y_{1}-y_{2}\right\|$ for any functions $y_{1}, y_{2}$ defined on $[v-3, v+b]_{\mathbb{Z}_{v-3}}$. Then if the condition

$$
2 \alpha \prod_{j=1}^{b}\left(\frac{v+j}{j}\right)+\beta<1
$$

holds, then problem (1.1) has a unique solution.

Proof We show that $T$ is a contraction mapping. To achieve this, we notice that for given $y_{1}$ and $y_{2}$,

$$
\begin{aligned}
& \left\|T y_{1}-T y_{2}\right\| \leq \alpha\left\|y_{1}-y_{2}\right\| \max _{t \in[v-3, v+b]_{\mathbb{Z}_{v-3}}}\left[\frac{1}{\Gamma(v)} \sum_{s=0}^{t-v}(t-s-1)^{\frac{v-1}{w}}\right] \\
& +\alpha\left\|y_{1}-y_{2}\right\| \max _{t \in[v-3, v+b]_{\mathbb{Z}_{v-3}}} \frac{t^{\frac{\nu-1}{}}}{\Gamma(v)(v+b)^{\nu-1}} \sum_{s=0}^{b}(v+b-s-1)^{\frac{\nu-1}{w}} \\
& +\beta\left\|y_{1}-y_{2}\right\| \max _{t \in[v-3, v+b]_{\mathbb{Z}_{v-3}}} \frac{t \frac{v-1}{(v+b) \frac{v-1}{}}}{(v .}
\end{aligned}
$$

By an application of Lemma 2.3, we get

$$
\begin{aligned}
\frac{1}{\Gamma(v)} \sum_{s=0}^{t-v}(t-s-1) \frac{v-1}{} & =\frac{1}{\Gamma(v)}\left[-\frac{1}{v}(t-s)^{\underline{v}}\right]_{s=0}^{t-v+1} \\
& =\frac{\Gamma(t+1)}{\Gamma(v+1) \Gamma(t+1-v)} \\
& \leq \frac{\Gamma(v+b+1)}{\Gamma(b+1) \Gamma(v+1)} \\
& =\prod_{j=1}^{b}\left(\frac{v+j}{j}\right) .
\end{aligned}
$$


Similar to the above inequality, we have

$$
\begin{aligned}
& \frac{t^{\nu-1}}{\Gamma(\nu)(\nu+b)^{\nu-1}} \sum_{s=0}^{b}(v+b-s-1)^{\frac{\nu-1}{}} \leq \frac{1}{\Gamma(\nu)} \sum_{s=0}^{b}(v+b-s-1)^{\frac{\nu-1}{v}} \\
& =\frac{1}{\Gamma(v)}\left[-\frac{1}{v}(v+b-s)^{\underline{v}}\right]_{s=0}^{b+1} \\
& =\frac{\Gamma(v+b+1)}{\Gamma(b+1) \Gamma(v+1)} \\
& =\prod_{j=1}^{b}\left(\frac{v+j}{j}\right) \text {. }
\end{aligned}
$$

From another application of Lemma 2.1, we obtain

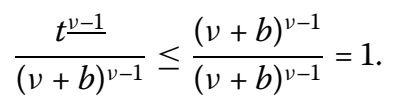

So, putting (3.4)-(3.6) in (3.3), we conclude that

$$
\left\|T y_{1}-T y_{2}\right\| \leq\left\{2 \alpha \prod_{j=1}^{b}\left(\frac{v+j}{j}\right)+\beta\right\}\left\|y_{1}-y_{2}\right\| .
$$

Then condition (3.2) holds. We find that (1.1) has a unique solution, which completes the proof of the theorem.

By weakening the conditions imposed on $f(t, y)$ and $g(y)$, we can still obtain the existence of a solution to (1.1). We apply the Brouwer theorem to accomplish this.

Theorem 3.2 Suppose that there exists a constant $K>0$ such that $f(t, y)$ satisfies the inequality

$$
\max _{(t, y) \in[v-3, v+b]_{\mathbb{Z}_{v-3}} \times[-K, K]}|f(t, y)| \leq \frac{K}{\frac{2 \Gamma(v+b+1)}{\Gamma(v+1) \Gamma(b+1)}+1}
$$

and $g(y)$ satisfies the inequality

$$
\max _{y \in[-K, K]}|g(y)| \leq \frac{K}{\frac{2 \Gamma(v+b+1)}{\Gamma(v+1) \Gamma(b+1)}+1} .
$$

Then (1.1) has at least one solution $y_{0}$ satisfying $\left|y_{0}(t)\right| \leq K$ for all $t \in[v-3, v+b]_{\mathbb{Z}_{v-3}}$.

Proof Consider the Banach space $\mathcal{B}:=\left\{y \in \mathbb{R}^{b+4}:\|y\| \leq K\right\}$. T is defined as (3.1). It is obvious that $T$ is a continuous operator. Therefore, our main objective is to show that $T: \mathscr{B} \rightarrow \mathcal{B}$. That is, whenever $\|y\| \leq K$, it follows that $\|T y\| \leq K$. Once this is established, we use the Brouwer theorem to deduce the conclusion.

Assume that inequalities (3.7) and (3.8) hold for given $f$ and $g$. For convenience, we let

$$
\Phi:=\frac{K}{\frac{2 \Gamma(v+b+1)}{\Gamma(v+1) \Gamma(b+1)}+1},
$$


which is a strictly positive constant. Then we have

$$
\begin{aligned}
& \|T y\| \leq \max _{t \in[v-3, v+b]_{\mathbb{Z}_{v-3}}} \frac{1}{\Gamma(v)} \sum_{s=0}^{t-v}(t-s-1)^{\frac{v-1}{}}|f(s+v-1, y(s+v-1))| \\
& +\max _{t \in[v-3, v+b]_{\mathbb{Z}_{v-3}}} \frac{t^{\frac{\nu-1}{}}}{\Gamma(v)(v+b)^{v-1}} \sum_{s=0}^{b}(v+b-s-1)^{\frac{v-1}{1}}|f(s+v-1, y(s+v-1))| \\
& +\max _{t \in[v-3, v+b]_{\mathbb{Z}_{\nu-3}}} \frac{t^{\nu-1}}{(v+b)^{\nu-1}}|g(y)| \\
& \leq \Phi \max _{t \in[\nu-3, v+b]_{\mathbb{Z}_{\nu-3}}}\left[\frac{1}{\Gamma(\nu)} \sum_{s=0}^{t-v}(t-s-1)^{\frac{\nu-1}{}}+\frac{t^{\nu-1}}{\Gamma(\nu)(v+b)^{\nu-1}} \sum_{s=0}^{b}(v+b-s-1)^{\frac{\nu-1}{}}\right]
\end{aligned}
$$

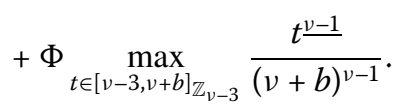

As in the proof of Theorem 3.1, we can simplify the expression on the right-hand side of inequality (3.10). Indeed, we know that

$$
\begin{aligned}
& \frac{1}{\Gamma(v)} \sum_{s=0}^{t-v}(t-s-1)^{\frac{\nu-1}{1}}+\frac{t^{\nu-1}}{\Gamma(v)(v+b)^{\nu-1}} \sum_{s=0}^{b}(v+b-s-1)^{\frac{\nu-1}{}} \\
& \leq \frac{1}{\Gamma(v)} \sum_{s=0}^{t-v}(t-s-1)^{\frac{\nu-1}{}}+\frac{1}{\Gamma(v)} \sum_{s=0}^{b}(v+b-s-1)^{\frac{\nu-1}{}} \\
& \leq \frac{1}{\Gamma(v)} \sum_{s=0}^{b}(v+b-s-1)^{\frac{\nu-1}{}}+\frac{1}{\Gamma(v)} \sum_{s=0}^{b}(v+b-s-1)^{\nu-1} \\
& =\frac{2}{\Gamma(\nu)} \sum_{s=0}^{b}(v+b-s-1)^{v-1} \text {. }
\end{aligned}
$$

On the one hand, from Lemma 2.1 we know $t \frac{\nu-1}{}$ is increasing in $t$, thus we have

$$
\sum_{s=0}^{b}(v+b-s-1)^{\frac{v-1}{v}}=\left[-\frac{1}{v}(v+b-s)^{\underline{v}}\right]_{s=0}^{b+1}=\frac{\Gamma(v+b+1)}{\nu \Gamma(b+1)} .
$$

On the other hand,

$$
\frac{t^{\nu-1}}{(v+b)^{\nu-1}} \leq 1
$$

Inserting (3.11)-(3.13) into (3.10), we can obtain

$$
\|T y\| \leq \Phi\left[\frac{2 \Gamma(v+b+1)}{\Gamma(v+1) \Gamma(b+1)}\right]+\Phi=\Phi\left[\frac{2 \Gamma(v+b+1)}{\Gamma(v+1) \Gamma(b+1)}+1\right] .
$$

By substituting (3.9) into (3.14), we have

$$
\|T y\| \leq \Phi\left[\frac{2 \Gamma(v+b+1)}{\Gamma(v+1) \Gamma(b+1)}+1\right]=K .
$$


Thus, from (3.15) we deduce that $T: \mathscr{B} \rightarrow \mathscr{B}$. Consequently, it follows at once by the Brouwer theorem that there exists a fixed point of the map $T$, say $T y_{0}=y_{0}$ with $y_{0} \in \mathcal{B}$. So, this function $y_{0}$ is a solution of (1.1) and $y_{0}$ satisfies the bound $\left|y_{0}(t)\right| \leq K$ for each $t \in[v-3, v+b]_{\mathbb{Z}_{v-3}}$. And this completes the proof of the theorem.

\section{Existence of a positive solution}

In this section, we show the existence of positive solutions for boundary value problem (1.2).

Lemma 4.1 [26] Let $\mathcal{B}$ be a Banach space, and let $\mathcal{K} \subseteq \mathcal{B}$ be a cone. Assume that $\Omega_{1}$ and $\Omega_{2}$ are two bounded open subsets contained in $\mathcal{B}$ such that $0 \in \Omega_{1}$ and $\bar{\Omega}_{1} \subseteq \Omega_{2}$. Assume further that $T: \mathcal{K} \cap\left(\bar{\Omega}_{2} \backslash \Omega_{1}\right) \rightarrow \mathcal{K}$ is a completely continuous operator. If either

(1) $\|T y\| \leq\|y\|$ for $y \in \mathcal{K} \cap \partial \Omega_{1}$ and $\|T y\| \geq\|y\|$ for $y \in \mathcal{K} \cap \partial \Omega_{2}$, or

(2) $\|T y\| \geq\|y\|$ for $y \in \mathcal{K} \cap \partial \Omega_{1}$ and $\|T y\| \leq\|y\|$ for $y \in \mathcal{K} \cap \partial \Omega_{2}$,

then $T$ has at least one fixed point in $\mathcal{K} \cap\left(\bar{\Omega}_{2} \backslash \Omega_{1}\right)$.

Define the Banach space $\mathcal{B}$ by

$$
\mathcal{B}=\left\{y:[v-3, v+b]_{\mathbb{Z}_{v-3}} \rightarrow \mathbb{R}: y(v-2)=y(v-3)=0, y(v+b)=g(y)\right\}
$$

with the norm $\|y\|=\max \left\{|y(t)|, t \in[v-3, v+b]_{\mathbb{Z}_{v-3}}\right\}$.

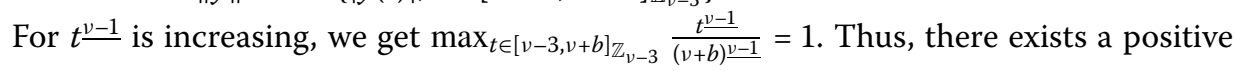
constant $\gamma_{0}$ such that

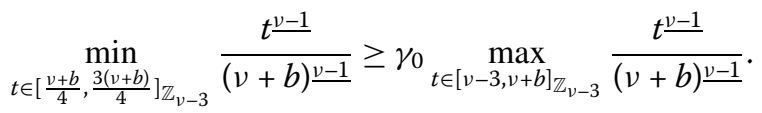

In fact, for $t \in\left[\frac{v+b}{4}, \frac{3(v+b)}{4}\right]_{\mathbb{Z}_{v-3}}, \frac{t^{\nu-1}}{(v+b)^{\underline{v-1}}}$ is strictly positive, then we let

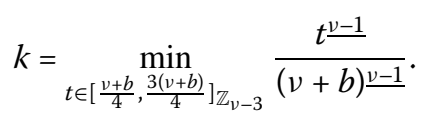

Take $0<\gamma_{0} \leq k$. Then

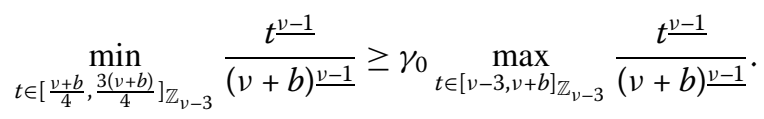

Denote $\tilde{\gamma}=\min \left\{\gamma, \gamma_{0}\right\}$. Then we have

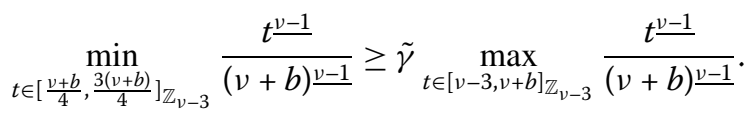

Define the cone

$$
\mathcal{P}=\left\{y \in \mathcal{B}: y(t) \geq 0, \min _{t \in\left[\frac{v+b}{4}, \frac{3(v+b)}{4}\right]_{\mathbb{Z}_{\nu-3}}} y(t) \geq \tilde{\gamma}\|y\|, t \in[v-3, v+b]_{\mathbb{Z}_{v-3}}\right\} .
$$


We define an operator $T_{\lambda}: \mathcal{P} \rightarrow \mathcal{B}$ as follows:

$$
\left(T_{\lambda} y\right)(t)=\lambda \sum_{s=0}^{b} G(t, s) f(s+v-1, y(s+v-1))+\lambda \frac{t^{\frac{v-1}{}}}{(v+b)^{\underline{v-1}}} g(y), \quad t \in[v-3, v+b]_{\mathbb{Z}_{v-3}} .
$$

It is easy to see from Lemma 2.4 that $y$ is a solution of (1.2) if and only if $y$ is a fixed point of $T_{\lambda}$.

Suppose that $f$ is a nonnegative function. Then, from Theorem 2.1 and (4.1), we have

$$
\begin{aligned}
& \min _{t \in\left[\frac{v+b}{4}, \frac{3(v+b)}{4}\right] \mathbb{Z}_{v-3}}\left(T_{\lambda} y\right)(t) \\
& =\min _{t \in\left[\frac{v+b}{4}, \frac{3(v+b)}{4}\right]_{\mathbb{Z}_{v-3}}} \lambda \sum_{s=0}^{b} G(t, s) f(s+v-1, y(s+v-1))
\end{aligned}
$$

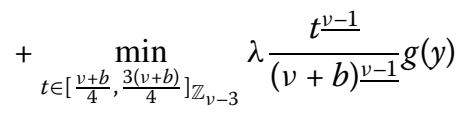

$$
\begin{aligned}
& \geq \gamma \lambda \max _{t \in[v-3, v+b]_{\mathbb{Z}_{v-3}}} \sum_{s=0}^{b} G(t, s) f(s+v-1, y(s+v-1)) \\
& +\tilde{\gamma} \lambda \max _{t \in[v-3, v+b]_{\mathbb{Z}_{\nu-3}}} \frac{t^{\underline{\nu-1}}}{(v+b) \underline{\underline{v-1}}} g(y) \\
& \geq \tilde{\gamma} \lambda \max _{t \in[v-3, v+b]_{\mathbb{Z}_{v-3}}} \sum_{s=0}^{b} G(t, s) f(s+\nu-1, y(s+v-1)) \\
& +\tilde{\gamma} \lambda \max _{t \in[v-3, v+b]_{\mathbb{Z}_{v-3}}} \frac{t^{\underline{\nu-1}}}{(v+b) \underline{\underline{v-1}}} g(y) \\
& =\tilde{\gamma}\left\|T_{\lambda} y\right\| .
\end{aligned}
$$

Thus, $T_{\lambda}(\mathcal{P}) \subset \mathcal{P}$.

Lemma 4.2 $T_{\lambda}: \mathcal{P} \rightarrow \mathcal{P}$ is completely continuous.

Proof Note that $T_{\lambda}$ is a summation operator on a discrete finite set, so $T_{\lambda}$ is trivially completely continuous.

For convenience, we define:

(F1) $f(t, y)=h(t) g(y)$, where $h$ is a positive function, $g$ is a nonnegative functional;

$$
\lim _{\|y\| \rightarrow 0^{+}} \frac{g(y)}{\|y\|}=0, \quad \lim _{\|y\| \rightarrow+\infty} \frac{g(y)}{\|y\|}=+\infty
$$

$$
\lim _{\|y\| \rightarrow 0^{+}} \frac{g(y)}{\|y\|}=+\infty, \quad \lim _{\|y\| \rightarrow+\infty} \frac{g(y)}{\|y\|}=0
$$


Set $l=\left\lfloor\frac{3(v+b)}{4}-v+1\right\rfloor+1-\left\lceil\frac{v+b}{4}-v+1\right\rceil, M=\max G(t, s)$ for $(t, s) \in[v-3, v+b]_{\mathbb{Z}_{v-3}} \times[0, b]$, $m=\sum_{s=\left\lceil\frac{v+b}{4}-v+1\right\rceil}^{\left\lfloor\frac{3(v+b)}{4}-v+1\right\rfloor} G(s+v-1, s), h=\min h(t), H=\max h(t)$ for $t \in[v-3, v+b]_{\mathbb{Z}_{v-3}}$.

Theorem 4.1 Suppose that conditions (F1) and (F2) hold. If there exist a sufficiently small positive constant $\delta$ and a sufficiently large constant $L_{1}$ such that $(M H(b+1)+1) \delta<m h L_{1}$ holds, then for each

$$
\lambda \in\left(\left(m h L_{1}\right)^{-1},((M H(b+1)+1) \delta)^{-1}\right),
$$

boundary value problem (1.2) has at least one positive solution.

Proof By condition (F2), there exists $r_{1}>0$ such that

$$
g(y) \leq \delta r_{1}, \quad 0<\|y\| \leq r_{1} .
$$

So, for $y \in \mathcal{P}$ with $\|y\|=r_{1}$, by (4.3) and (4.4), we have, for all $t \in[v-3, v+b]_{\mathbb{Z}_{\nu-3}}$,

$$
\begin{aligned}
\left(T_{\lambda} y\right)(t) & =\lambda \sum_{s=0}^{b} G(t, s) f(s+v-1, y(s+v-1))+\lambda \frac{t \frac{v-1}{(v+b) \frac{v-1}{y}} g(y)}{\leq} \\
& \leq \lambda M \sum_{s=0}^{b} h(s+v-1) g(y(s+v-1))+\lambda g(y) \\
& \leq \lambda M H(b+1) \delta r_{1}+\lambda \delta r_{1} \\
& =\lambda(M H(b+1)+1) \delta r_{1} \\
& \leq r_{1}=\|y\| .
\end{aligned}
$$

Thus, if we choose $\Omega_{1}=\left\{y \in \mathcal{B}:\|y\|<r_{1}\right\}$, then (4.5) implies that

$$
\left\|T_{\lambda} y\right\| \leq\|y\|, \quad y \in \mathcal{P} \cap \partial \Omega_{1} .
$$

Similarly, by condition (F2), we can find $0<r_{1}<r_{2}$ and a sufficiently large constant $L_{1}$ such that

$$
g(y) \geq \frac{L_{1}}{\gamma^{2}} r_{2}, \quad\|y\| \geq r_{2} .
$$

And then we set $r_{2}^{*}=r_{2} / \gamma>r_{2}$ and $\Omega_{2}=\left\{y \in \mathcal{B}:\|y\|<r_{2}^{*}\right\}$. Then $y \in \mathcal{P}$ and $\|y\|=r_{2}^{*}$ imply

$$
\min _{t \in\left[\frac{v+b}{4}, \frac{3(v+b)}{4}\right]_{\mathbb{Z}_{\nu-2}}} y(t) \geq \gamma\|y\|=\gamma r_{2}^{*}=r_{2}
$$

thus

$$
y(t) \geq r_{2} \quad \text { for all } t \in\left[\frac{v+b}{4}, \frac{3(v+b)}{4}\right]_{\mathbb{Z}_{v-3}} .
$$


Therefore, for given $t_{0} \in\left[\frac{b+v}{4}, \frac{3(b+v)}{4}\right]$, by Theorem 2.1, (4.3) and (4.7), we have

$$
\begin{aligned}
\left(T_{\lambda} y\right)\left(t_{0}\right) & =\lambda \sum_{s=0}^{b} G\left(t_{0}, s\right) f(s+v-1, y(s+v-1))+\lambda \frac{t \frac{v-1}{(v+b) \frac{v-1}{2}} g(y)}{} \\
& \geq \lambda \sum_{s=\left\lceil\frac{\left.\frac{v+b}{4}-v+1\right\rceil}{4}-v+1\right\rfloor}^{\left\lfloor\frac{3(+b)}{4}\right.} G\left(t_{0}, s\right) h(s+v-1) g(y(s+v-1)) \\
& \geq \lambda \gamma h \frac{L_{1}}{\gamma^{2}} r_{2} \sum_{s=\left\lceil\frac{v+b}{4}-v+1\right\rceil}^{\left\lfloor\frac{3(v+b)}{4}-v+1\right\rfloor} G(s+v-1, s) \\
& \geq \lambda m h L_{1} r_{2}^{*} \\
& \geq r_{2}^{*}=\|y\| .
\end{aligned}
$$

Hence, from (4.8) we have

$$
\left\|T_{\lambda} y\right\| \geq\|y\|, \quad y \in \mathcal{P} \cap \partial \Omega_{2} .
$$

Now, from (4.6), (4.9) and Lemma 4.1, we have $T_{\lambda}$ has a fixed point $y \in \mathcal{P} \cap\left(\bar{\Omega}_{2} \backslash \Omega_{1}\right)$ with $r_{1} \leq\|y\| \leq r_{2}^{*}$. Then the theorem is proved.

Theorem 4.2 Assume that conditions (F1) and (F3) hold. If there exists a sufficiently large constant $L_{2}$ such that $(M b H+1)<m h L_{2}$ holds, then for each

$$
\lambda \in\left(\left(m h L_{2}\right)^{-1},(M H(b+1)+1)^{-1}\right),
$$

boundary value problem (1.2) has at least one positive solution.

Proof By condition (F3), there exist $r_{3}>0$ and a sufficiently large constant $L_{2}>0$ such that

$$
g(y)>\frac{L}{\gamma} r_{3} \quad \text { for } 0<\|y\|<r_{3}
$$

Let $\Omega_{1}=\left\{y \in \mathcal{B},\|y\|<r_{3}\right\}$. Then, for $y \in \Omega_{1}$,

$$
\begin{aligned}
\left(T_{\lambda} y\right)(t) & =\lambda \sum_{s=0}^{b} G(t, s) f(s+v-1, y(s+v-1))+\lambda \frac{t \frac{v-1}{(v+b) \underline{v-1}}}{(y)} \\
& \geq \lambda \sum_{s=\left\lceil\frac{v+b}{4}-v+1\right\rceil}^{\left\lfloor\frac{3(v+b)}{4}-v+1\right\rfloor} G(t, s) h(s+v-1) g(y(s+v-1)) \\
& \geq \lambda \gamma m h \frac{L_{2}}{\gamma} r_{3} \\
& =\lambda m h L_{2} r_{3} \\
& \geq r_{3}=\|y\| .
\end{aligned}
$$


Then (4.12) implies that

$$
\left\|T_{\lambda} y\right\| \geq\|y\|, \quad y \in \mathcal{P} \cap \partial \Omega_{1}
$$

Next, we consider two cases for the construction of $\Omega_{2}$.

Case 1. Suppose that $g$ is bounded. Then there exists some $R_{1}>r_{3}$ such that

$$
g(y) \leq R_{1} \quad \text { for } y \in \mathcal{P} .
$$

From (4.10) we know

$$
\lambda<(M H(b+1)+1)^{-1}
$$

Thus, from (4.14) and (4.15), we get

$$
\begin{aligned}
\left(T_{\lambda} y\right)(t) & =\lambda \sum_{s=0}^{b} G(t, s) f(s+v-1, y(s+v-1))+\lambda \frac{t \frac{v-1}{(v+b) \underline{v-1}}}{(y)} \\
& \leq \lambda M \sum_{s=0}^{b} h(s+v-1) g(y(s+v-1))+\lambda g(y) \\
& \leq \lambda M H R_{1}(b+1)+\lambda R_{1} \\
& =\lambda(M H(b+1)+1) R_{1} \\
& \leq R_{1}=\|y\| .
\end{aligned}
$$

Case 2. Suppose that $g$ is unbounded. From (4.10) we know $\lambda<(M H(b+1)+1)^{-1}$, so $\lambda<\left((M H(b+1)+1) \delta_{2}\right)^{-1}$ for a sufficiently small constant $\delta_{2}$. Then, by condition (F3), there exists some $R_{2}$ such that

$$
g(y) \leq \delta_{2}\|y\|, \quad\|y\| \geq R_{2} .
$$

Choose $R_{3}$ such that $R_{3}>r_{3}$ and for $0<\|y\| \leq R_{3}, g(y) \leq g\left(R_{3}\right)$. Define $R=\max \left\{R_{2}, R_{3}\right\}$. Now we set $\Omega_{2}=\{y \in \mathcal{B}:\|y\|<R\}$, then $g(R) \leq \delta_{2} R$. Thus, for $y \in \partial \Omega_{2}$, we have

$$
\begin{aligned}
\left(T_{\lambda} y\right)(t) & =\lambda \sum_{s=0}^{b} G(t, s) f(s+v-1, y(s+v-1))+\lambda \frac{t^{\frac{v-1}{(v+b)}}}{(v-1} g(y) \\
& \leq \lambda M \sum_{s=0}^{b} h(s+v-1) g(y(s+v-1))+\lambda g(y) \\
& \leq \lambda M H(b+1) \delta_{2} R+\lambda \delta_{2} R \\
& =\lambda(M H(b+1)+1) \delta_{2} R \\
& \leq R=\|y\| .
\end{aligned}
$$

Then, in both Case 1 and Case 2, we have

$$
\left\|T_{\lambda} y\right\| \leq\|y\|, \quad y \in \mathcal{P} \cap \partial \Omega_{2} .
$$


From (4.13), (4.19) and Lemma 4.1, we get $T_{\lambda}$ has a fixed point $y \in \mathcal{P} \cap\left(\bar{\Omega}_{2} \backslash \Omega_{1}\right)$ with $r_{3} \leq\|y\| \leq R$. This completes the proof.

\section{Nonexistence}

In this section, we give some sufficient conditions for the nonexistence of a positive solution to boundary value problem (1.2).

We state the following hypotheses that will be used in what follows.

(F4)

$$
g_{0}=\limsup _{\|y\| \rightarrow 0^{+}} \frac{g(y)}{\|y\|}, \quad g_{\infty}=\limsup _{\|y\| \rightarrow+\infty} \frac{g(y)}{\|y\|}
$$

$$
g_{0}^{*}=\liminf _{\|y\| \rightarrow 0^{+}} \frac{g(y)}{\|y\|}, \quad g_{\infty}^{*}=\liminf _{\|y\| \rightarrow+\infty} \frac{g(y)}{\|y\|} .
$$

Theorem 5.1 Assume that (F1) and (F4) hold. If $g_{0}<+\infty$ and $g_{\infty}<+\infty$, then there exists $\lambda_{0}$ such that for all $0<\lambda<\lambda_{0}$, boundary value problem (1.2) has no positive solution.

Proof Since $g_{0}<+\infty$ and $g_{\infty}<+\infty$, there exist positive numbers $c_{1}, c_{2}, r_{1}$ and $r_{2}$ such that $r_{1}<r_{2}$ and

$$
\begin{array}{ll}
g(y) \leq c_{1}\|y\| & \text { for }\|y\| \in\left[0, r_{1}\right], \\
g(y) \leq c_{2}\|y\| & \text { for }\|y\| \in\left[r_{2},+\infty\right) .
\end{array}
$$

Let $c=\max \left\{c_{1}, c_{2}, \max _{r_{1} \leq\|y\| \leq r_{2}} \frac{g(y)}{\|y\|}\right\}$. Then we have

$$
g(y) \leq c\|y\| \quad \text { for all }\|y\| \in[0,+\infty)
$$

Suppose that $y_{0}(t)$ is a positive solution of (1.2). Then we show that this leads to a contradiction for $0<\lambda<\lambda_{0}:=((M H(b+1)+1) c)^{-1}$. Since $T_{\lambda} y_{0}(t)=y_{0}(t)$, for $t \in[v-3, v+b]_{\mathbb{Z}_{v-3}}$,

$$
\left\|y_{0}\right\|=\left\|T_{\lambda} y_{0}\right\| \leq \lambda M H \sum_{s=1}^{b} g\left(y_{0}(s+v-1)\right)+\lambda g\left(y_{0}\right) \leq \lambda(M H(b+1)+1) c\left\|y_{0}\right\|<\left\|y_{0}\right\|,
$$

which is a contradiction. Therefore, (1.2) has no positive solution. This completes the proof.

Theorem 5.2 Assume that (F1) and (F5) hold. If $g_{0}^{*}>0$ and $g_{\infty}^{*}>0$, then there exists $\lambda_{0}$ such that for all $\lambda>\lambda_{0}$, boundary value problem (1.2) has no positive solution.

Proof Since $g_{0}^{*}>0$ and $g_{\infty}^{*}>0$, we can get that there exist positive numbers $\eta_{1}, \eta_{2}, r_{1}, r_{2}$ such that $r_{1}<r_{2}$, and

$$
\begin{aligned}
& g(y) \geq \eta_{1}\|y\|, \quad\|y\| \in\left[0, r_{1}\right], \\
& g(y) \geq \eta_{2}\|y\|, \quad\|y\| \in\left[r_{2},+\infty\right) .
\end{aligned}
$$


Let $\eta=\min \left\{\eta_{1}, \eta_{2}, \min _{r_{1} \leq\|y\| \leq r_{2}} \frac{g(y)}{\|y\|}\right\}>0$. Then

$$
g(y) \geq \eta\|y\| \quad \text { for }\|y\| \in[0,+\infty) .
$$

Assume that $y_{1}(t)$ is a positive solution of (1.2). We show that this leads to a contradiction for $\lambda>\lambda_{0}:=(\gamma m H l \eta)^{-1}$. Since $T_{\lambda} y_{1}(t)=y_{1}(t)$, for $t \in[v-3, v+b]_{\mathbb{Z}_{v-3}}$, thus

$$
\begin{aligned}
\left\|y_{1}\right\| & =\left\|T_{\lambda} y_{1}\right\| \geq \lambda \sum_{s=\left\lceil\frac{v+b}{4}-v+1\right\rceil}^{\left\lfloor\frac{3(v+b)}{4}-v+1\right\rfloor} G(t, s) h(s+v-1) g(y(s+v-1)) \\
& \geq \lambda \gamma m h \eta\left\|y_{1}\right\|>\left\|y_{1}\right\|,
\end{aligned}
$$

which is a contradiction. Thus, (1.2) has no positive solution. The proof is completed.

\section{Example}

In this section, we present some examples to illustrate the main results.

Example 6.1 Suppose that $v=\frac{5}{2}, b=8$. Let $f(t, y(t)):=\frac{|\cos y(t)|}{1,000+t^{2}}$ and $g(y):=\frac{1}{20}\|\sin y\|$. Then (1.1) becomes

$$
\left\{\begin{array}{l}
-\Delta^{\frac{5}{2}} y(t)=\frac{|\cos y(t)|}{1,000+t^{2}}, \quad t=0,1,2, \ldots, 8, \\
y\left(-\frac{1}{2}\right)=0, \\
\Delta y\left(-\frac{1}{2}\right)=0 \\
y\left(\frac{21}{2}\right)=\frac{1}{20}\|\sin y\| .
\end{array}\right.
$$

In this case, let $\alpha=\frac{1}{500}, \beta=\frac{1}{20}$. Inequality (3.2) is

$$
2 \alpha \prod_{j=1}^{b}\left(\frac{v+j}{j}\right)+\beta \leq \frac{165}{250}+\frac{1}{20}<1 .
$$

Therefore, from Theorem 3.1 we deduce that problem (6.1) has a unique solution.

Example 6.2 Suppose that $v=\frac{5}{2}, b=8$ and $K=1,000$. Let $f(t, y):=\frac{1}{21} t \exp \left\{-\frac{1}{10} y^{2}\right\}$ and $g(y):=10\|\cos y\|$. Then problem (1.1) is

$$
\left\{\begin{array}{l}
-\Delta^{\frac{5}{2}} y(t)=5 t \exp \left\{-\frac{1}{10} y^{2}(t)\right\}, \quad t=0,1,2, \ldots, 8 \\
y\left(-\frac{1}{2}\right)=0 \\
\Delta y\left(-\frac{1}{2}\right)=0 \\
y\left(\frac{21}{2}\right)=10\|\cos y\| .
\end{array}\right.
$$

The Banach space $\mathcal{B}:=\left\{y \in \mathbb{R}^{12}:\|y\| \leq 1,000\right\}$.

We note that

$$
\frac{K}{\frac{2 \Gamma(v+b+1)}{\Gamma(v+1) \Gamma(b+1)}+1}=\frac{1,000}{\frac{2 \Gamma\left(\frac{23}{2}\right)}{\Gamma\left(\frac{7}{2}\right) \Gamma(9)}+1}>\frac{1,000}{970} \approx 10.3 .
$$


It is clear that $|f(t, y)| \leq 0.5<10.3,|g(y)| \leq 10<10.3$. So, $f$ and $g$ satisfy the conditions. Thus, by Theorem 3.2 we deduce that problem (6.2) has at least one solution.

Example 6.3 Suppose that $v=\frac{5}{2}, b=8$. Let $h(t)=2 t$ for $t \in[v-1, v+b]_{\mathbb{Z}_{v-1}}, g(y)=\left\|y^{3}\right\|$ for $\|y\| \in(0,+\infty)$. Take $L_{1}=400, \delta=\frac{1}{50,000}$. Then $f(t, y)=2 t y^{3}$, and problem (1.2) becomes

$$
\left\{\begin{array}{l}
-\Delta^{\frac{5}{2}} y(t)=2 \lambda t y^{3}(t), \quad t \in[0,8]_{\mathbb{Z}} \\
y\left(-\frac{1}{2}\right)=0 \\
\Delta y\left(-\frac{1}{2}\right)=0 \\
y\left(\frac{21}{2}\right)=\left\|y^{3}\right\| .
\end{array}\right.
$$

By routine numerical calculations, we have

$$
\begin{aligned}
& M=\max _{(t, s) \in[v-3, v+b]_{\mathbb{Z}_{v-3}} \times[0, b]} G(t, s) \approx 5.1454, \\
& H=2(v+b)=2\left(\frac{5}{2}+8\right)=21, \quad h=2(v-1)=2 \times \frac{3}{2}=3, \\
& m=\sum_{s=\left\lceil\frac{v+b}{4}-v+1\right\rceil}^{\left\lfloor\frac{3(v+b)}{4}-v+1\right\rfloor} G(s+v-1, s)=\sum_{s=2}^{6} G(s+v-1, s) \approx 8.5318
\end{aligned}
$$

and

$$
\lim _{\|y\| \rightarrow 0^{+}} \frac{g(y)}{\|y\|}=0, \quad \lim _{\|y\| \rightarrow+\infty} \frac{g(y)}{\|y\|}=+\infty .
$$

Then

$$
\begin{aligned}
& (M H(b+1)+1) \delta=(5.1454 \times 21 \times 9+1) \times \frac{1}{50,000}=0.0195, \\
& m h L_{1}=8.5318 \times 3 \times 400=10,238.16
\end{aligned}
$$

thus $(M H b+1) \delta<m h L_{1}$. So, the conditions of Theorem 4.1 are satisfied. Since

$$
((M H(b+1)+1) \delta)^{-1}=\frac{1}{0.0195} \approx 51.2821, \quad\left(m h L_{1}\right)^{-1} \approx 0.0001,
$$

thus by Theorem 4.1 we have that boundary value problem (6.3) has at least one positive solution for each $\lambda \in(0.0001,51.2821)$.

Example 6.4 Suppose that $v=\frac{5}{2}, b=8$. Let $h(t)=\frac{1}{500} t$ for $t \in[v-1, v+b]_{\mathbb{Z}_{v-1}}, g(y)=e^{-\|y\|}$ for $y \in(0,+\infty)$. Take $L_{2}=1,000$. Then $f(t, y)=\frac{1}{500} t e^{-y}$, and problem (1.2) becomes

$$
\left\{\begin{array}{l}
-\Delta^{\frac{5}{2}} y(t)=\frac{1}{500} \lambda t e^{-y(t)}, \quad t \in[0,8]_{\mathbb{Z}}, \\
y\left(-\frac{1}{2}\right)=0 \\
\Delta y\left(-\frac{1}{2}\right)=0 \\
y\left(\frac{21}{2}\right)=e^{-\|y\|}
\end{array}\right.
$$


By routine numerical calculations, we have

$$
\begin{aligned}
& M=\max _{(t, s) \in[v-3, v+b]_{\mathbb{Z}_{\nu-3}} \times[0, b]} G(t, s) \approx 5.1454, \\
& H=\frac{1}{500}(v+b)=\frac{1}{500}\left(\frac{5}{2}+8\right)=\frac{21}{1,000}, \quad h=\frac{1}{500}(v-1)=\frac{1}{500} \times \frac{3}{2}=\frac{3}{1,000}
\end{aligned}
$$

and

$$
\lim _{\|y\| \rightarrow 0^{+}} \frac{g(y)}{\|y\|}=\lim _{\|y\| \rightarrow 0^{+}} \frac{e^{-\|y\|}}{\|y\|}=+\infty, \quad \lim _{\|y\| \rightarrow+\infty} \frac{g(y)}{\|y\|}=\lim _{\|y\| \rightarrow+\infty} \frac{e^{-\|y\|}}{\|y\|}=0 .
$$

Then

$$
\begin{aligned}
& M H(b+1)+1=\left(5.1454 \times \frac{21}{1,000} \times 9+1\right) \approx 1.9724 \\
& m h L_{2}=8.5318 \times \frac{3}{1,000} \times 1,000=25.5954
\end{aligned}
$$

thus $M H(b+1)+1<m h L_{2}$. So, the conditions of Theorem 4.2 are satisfied. Since

$$
(M H(b+1)+1)^{-1}=\frac{1}{1.9724} \approx 0.507, \quad\left(m h L_{2}\right)^{-1} \approx 0.0391
$$

then by Theorem 4.2 we deduce that boundary value problem (6.4) has at least one positive solution for each $\lambda \in(0.0391,0.507)$.

Example 6.5 Suppose that $v=\frac{5}{2}, b=8$. Let $h(t)=2 t$ for $t \in[v-1, v+b]_{\mathbb{Z}_{v-1}}, g(y)=y$ for $y \in(0,+\infty)$. Take $c_{1}=c_{2}=2$. Then $f(t, y)=2 t y$, and problem (1.2) becomes

$$
\left\{\begin{array}{l}
-\Delta^{\frac{5}{2}} y(t)=2 \lambda \operatorname{ty}(t), \quad t \in[0,8]_{\mathbb{Z}}, \\
y\left(-\frac{1}{2}\right)=0, \\
\Delta y\left(-\frac{1}{2}\right)=0 \\
y\left(\frac{21}{2}\right)=\|y\| .
\end{array}\right.
$$

Thus, we have

$$
g_{0}=\lim _{\|y\| \rightarrow 0^{+}} \sup \frac{g(y)}{\|y\|}=1<+\infty, \quad g_{\infty}=\lim _{\|y\| \rightarrow+\infty} \sup \frac{g(y)}{\|y\|}=1<+\infty
$$

By calculation,

$$
\begin{aligned}
& M=\max _{(t, s) \in[v-3, v+b]_{\mathbb{Z}_{v-3}} \times[0, b]} G(t, s) \approx 5.1454, \\
& H=2(v+b)=2\left(\frac{5}{2}+8\right)=21, \quad c=\max \left\{c_{1}, c_{2}, \max _{r_{1} \leq y \leq r_{2}} \frac{g(y)}{y}\right\}=2,
\end{aligned}
$$

so

$$
\lambda_{0}=((M H(b+1)+1) c)^{-1}=((5.1454 \times 21 \times 9+1) \times 2)^{-1} \approx 0.0005 .
$$

Therefore, by Theorem 5.1 we deduce that (6.5) has no positive solution for $0<\lambda<\lambda_{0}$. 
Example 6.6 Suppose that $v=\frac{5}{2}, b=8$. Let $h(t)=\frac{1}{1,050} t$ for $t \in[v-1, v+b]_{\mathbb{Z}_{v-1}}, g(y)=\|y\|$ for $y \in(0,+\infty)$. Take $\eta_{1}=\eta_{2}=\frac{1}{2}$. Then $f(t, y)=\frac{1}{1,050} t y$, and problem (1.2) becomes

$$
\left\{\begin{array}{l}
-\Delta^{\frac{5}{2}} y(t)=2 \lambda t y(t), \quad t \in[0,8]_{\mathbb{Z}} \\
y\left(-\frac{1}{2}\right)=0 \\
\Delta y\left(-\frac{1}{2}\right)=0 \\
y\left(\frac{21}{2}\right)=\|y\|
\end{array}\right.
$$

Thus, we have

$$
g_{0}=\lim _{\|y\| \rightarrow 0^{+}} \sup \frac{g(y)}{\|y\|}=1<+\infty, \quad g_{\infty}=\lim _{\|y\| \rightarrow+\infty} \sup \frac{g(y)}{\|y\|}=1<+\infty .
$$

By calculation,

$$
\begin{aligned}
& M=\max _{(t, s) \in[v-3, v+b]_{\mathbb{Z}_{v-3}} \times[0, b]} G(t, s) \approx 5.1454, \\
& H=\frac{1}{1,050}(v+b)=\frac{1}{1,050}\left(\frac{5}{2}+8\right)=\frac{1}{100}, \quad \eta=\min \left\{\eta_{1}, \eta_{2}, \min _{r_{1} \leq y \leq r_{2}} \frac{g(y)}{y}\right\}=\frac{1}{2}, \\
& l=\left\lfloor\frac{3(v+b)}{4}-v+1\right\rfloor+1-\left[\frac{v+b}{4}-v+1\right\rceil=\frac{25}{4} .
\end{aligned}
$$

By the definition of [14], we know

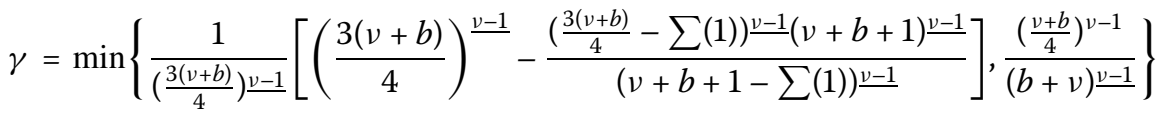

$$
\begin{aligned}
& \approx 0.518 \text {, }
\end{aligned}
$$

and then

$$
\lambda_{0}:=(\gamma M H l \eta)^{-1}=\left(\gamma M \times \frac{1}{100} \times \frac{25}{4} \times \frac{1}{2}\right)^{-1} \approx 12.01 .
$$

Hence by Theorem 5.2 we deduce that (6.6) has no positive solution for $\lambda>\lambda_{0}$.

\section{Conclusion}

This paper is an extension of [14] and [18]. The main contributions of this paper include:

- The existence and uniqueness of a solution to a class of boundary value problems for a fractional difference equation with $3<\alpha \leq 4$ are studied by the contraction mapping theorem.

- The existence of a solution to a class of boundary value problems for a fractional difference equation with $3<\alpha \leq 4$ is studied by the Brouwer fixed point theorem.

- The eigenvalue intervals of a boundary value problem for a class nonlinear fractional difference equations with $3<\alpha \leq 4$ are investigated by the Guo-Krasnosel'skii fixed point theorem.

- The nonexistence of a positive solution boundary value problem for a class nonlinear fractional difference equations with $3<\alpha \leq 4$ is considered in terms of parameter. 
In contrast to [14] and [18], the similarities and differences are as follows:

- The methods used to prove the existence results are standard and the same; however, their exposition in the framework of problems (1.1) and (1.2) is new.

- The major difference is that the equations have different fractional order. The order is $3<\alpha \leq 4$ in this paper and $1<\alpha \leq 2$ in [14] and [18]. The higher order leads the comparable process to being more difficult and complex.

- Nonlocal boundary conditions are considered in this paper and [18], Dirichlet boundary conditions are considered in [14].

- Both the existence and nonexistence are considered in this paper, but only the existence is considered in [14] and [18].

\section{Competing interests}

The authors declare that they have no competing interests.

\section{Authors' contributions}

The authors declare that the study was realized in collaboration with the same responsibility. All authors read and approved the final manuscript.

\section{Author details}

'School of Mathematical Sciences, University of Jinan, Jinan, Shandong 250022, P.R. China. ${ }^{2}$ Department of Mathematics and Statistics, Missouri University of Science and Technology, Rolla, Missouri 65409-0020, USA.

\section{Acknowledgements}

The authors sincerely thank the reviewers for their valuable suggestions and useful comments that have led to the present improved version of the original manuscript. This research is supported by the Natural Science Foundation of China (11071143), Natural Science Outstanding Youth Foundation of Shandong Province (JQ201119) and supported by Shandong Provincial Natural Science Foundation (ZR2012AM009), also supported by the Natural Science Foundation of Educational Department of Shandong Province (J11LA01).

Received: 4 February 2013 Accepted: 19 August 2013 Published: 19 September 2013

\section{References}

1. Podlubny, I: Fractional Differential Equations. Mathematics in Science and Engineering. Academic Press, New York (1999)

2. Bai, Z, Lü, H: Positive solutions for boundary value problem of nonlinear fractional differential equation. J. Math. Anal. Appl. 311, 495-505 (2005)

3. Benchohra, M, Hamani, S, Ntouyas, S: Boundary value problems for differential equations with fractional order and nonlocal conditions. Nonlinear Anal. TMA 71, 2391-2396 (2009)

4. Diethelm, K, Ford, N: Analysis of fractional differential equations. J. Math. Anal. Appl. 265, 229-248 (2002)

5. $\mathrm{Xu}, \mathrm{X}$, Jiang, $\mathrm{D}$, Yuan, $\mathrm{C}$ : Multiple positive solutions for the boundary value problem of a nonlinear fractional differential equation. Nonlinear Anal. TMA 71, 4676-4688 (2009)

6. Zhao, Y, Sun, S, Han, Z, Li, Q: The existence of multiple positive solutions for boundary value problems of nonlinear fractional differential equations. Commun. Nonlinear Sci. Numer. Simul. 16, 2086-2097 (2011)

7. Zhao, Y, Sun, S, Han, Z, Li, Q: Positive solutions to boundary value problems of nonlinear fractional differential equations. Abstr. Appl. Anal. 2011, Article ID 390543 (2011)

8. Zhao, Y, Sun, S, Han, Z, Li, Q: Theory of fractional hybrid differential equations. Comput. Math. Appl. 62, 1312-1324 (2011)

9. Pan, $Y$, Han, Z: Existence of solutions for a coupled system of boundary value problem of nonlinear fractional differential equations. In: Proceedings of the 5th International Congress on Mathematical Biology, vol. 1, pp. 109-114 (2011)

10. Zhou, Y, Jiao, F, Li, J: Existence and uniqueness for $p$-type fractional neutral differential equations. Nonlinear Anal. TMA 71(7-8), 2724-2733 (2009)

11. Zhou, Y, Jiao, F, Li, J: Existence and uniqueness for fractional neutral differential equations with infinite delay. Nonlinear Anal. TMA 71(7-8), 3249-3256 (2009)

12. Zhou, Y, Jiao, F: Nonlocal Cauchy problem for fractional evolution equations. Nonlinear Anal., Real World Appl. 11 4465-4475 (2010)

13. Atici, F, Eloe, P: A transform method in discrete fractional calculus. Int. J. Differ. Equ. 2, 165-176 (2007)

14. Atici, F, Eloe, P: Two-point boundary value problems for finite fractional difference equations. J. Differ. Equ. Appl. 17, 445-456 (2011)

15. Atici, F, Eloe, P: Initial value problems in discrete fractional calculus. Proc. Am. Math. Soc. 137(3), 981-989 (2009)

16. Goodrich, C: Solutions to a discrete right-focal fractional boundary value problem. Int. J. Differ. Equ. 5, 195-216 (2010)

17. Goodrich, C: Continuity of solutions to discrete fractional initial value problems. Comput. Math. Appl. 59, 3489-3499 (2010)

18. Goodrich, C: Existence and uniqueness of solutions to a fractional difference equation with nonlocal conditions. Comput. Math. Appl. 61, 191-202 (2011) 
19. Goodrich, C: On positive solutions to nonlocal fractional and integer-order difference equations. Appl. Anal. Discrete Math. 5, 122-132 (2011)

20. Goodrich, C: On discrete sequential fractional boundary value problems. J. Math. Anal. Appl. 385, 111-124 (2012)

21. Pan, Y, Han, Z, Sun, S, Zhao, Y: The existence of solutions to a system of discrete fractional boundary value problems. Abstr. Appl. Anal. (2012). doi:10.1155/2012/707631

22. Ferreira, R: Positive solutions for a class of boundary value problems with fractional $q$-differences. Comput. Math. Appl. 61, 367-373 (2011)

23. Bastos, N, et al.: Discrete-time fractional variational problems. Signal Process. 91, 513-524 (2011)

24. Atici, F, Sengül, S: Modeling with fractional difference equations. J. Math. Anal. Appl. 369, 1-9 (2010)

25. Atici, F, Eloe, P: Fractional q-calculus on a time scale. J. Nonlinear Math. Phys. 14(3), 333-344 (2007)

26. Agarwal, R, Meehan, M, O'Regan, D: Fixed Point Theory and Applications. Cambridge University Press, Cambridge (2001)

27. Goodrich, C: Existence of a positive solution to a system of discrete fractional boundary value problems. Appl. Math. Comput. 217, 4740-4753 (2011)

28. Henderson, J, Ntouyas, S, Purnaras, I: Positive solutions for systems of nonlinear discrete boundary value problem. J. Differ. Equ. Appl. 15, 895-912 (2009)

29. Dunninger, D, Wang, H: Existence and multiplicity of positive solutions for elliptic systems. Nonlinear Anal. TMA 29, 1051-1060 (1997)

30. Pan, Y, Han, Z, Sun, S, Huang, Z: The existence and uniqueness of solutions to boundary value problems of fractional difference equations. Math. Sci. 6(7), 1-7 (2012)

doi:10.1186/1687-1847-2013-275

Cite this article as: Pan et al.: The existence of solutions to a class of boundary value problems with fractional

difference equations. Advances in Difference Equations 2013 2013:275.

\section{Submit your manuscript to a SpringerOpen ${ }^{\ominus}$ journal and benefit from:}

- Convenient online submission

- Rigorous peer review

- Immediate publication on acceptance

- Open access: articles freely available online

- High visibility within the field

- Retaining the copyright to your article 